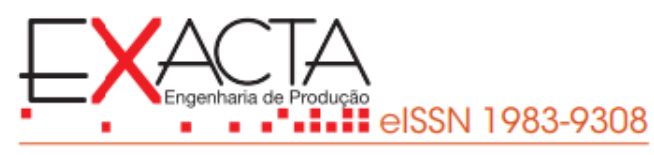

\title{
PROCESSO DE INOVAÇÃO EM EMPRESAS DE TECNOLOGIA: UM OLHAR SOBRE A CONTRIBUIÇÃO DOS SOFTWARES SOCIAIS E DAS FERRAMENTAS COLABORATIVAS
}

\author{
INNOVATION PROCESS IN TECHNOLOGY COMPANIES: AN ANALYSIS ON THE \\ CONTRIBUTION OF SOCIAL SOFTWARE AND COLLABORATIVE TOOLS
}

Versão do autor aceita publicada online: 13 fev. 2020

Publicado online: 12 maio 2021

Como citar esse artigo - American Psychological Association (APA):

Miranda, M. A. S., Santos, E. M., Albuquerque, A. E. Junior, Moraes, M. B., Ribeiro, E. M., \& Fonseca, P. G. (2021, abr./jun.). Processo de inovação em empresas de tecnologia: um olhar sobre a contribuição dos softwares sociais e das ferramentas colaborativas. Exacta, 19(2), 276301. https://doi.org/10.5585/exactaep.2021.13176.

Submeta seu artigo para este periódico $\beta$

Dados Crossmark 


\title{
PROCESSO DE INOVAÇÃO EM EMPRESAS DE TECNOLOGIA: UM OLHAR SOBRE A CONTRIBUIÇÃO DOS SOFTWARES SOCIAIS E DAS FERRAMENTAS COLABORATIVAS
}

\section{INNOVATION PROCESS IN TECHNOLOGY COMPANIES: AN ANALYSIS ON THE CONTRIBUTION OF SOCIAL SOFTWARE AND COLLABORATIVE TOOLS}

\author{
iD Morjane Armstrong Santos de Miranda ${ }^{1}$ \\ iD Ernani Marques dos Santos ${ }^{2}$ \\ (iD) Antonio Eduardo de Albuquerque Junior ${ }^{3}$ \\ (iD) Marcela Barbosa de Moraes ${ }^{4}$ \\ (iD) Elizabeth Matos Ribeiro ${ }^{5}$ \\ iD Platini Gomes Fonseca ${ }^{6}$
}

${ }^{1}$ Doutorado

Universidade Federal da Bahia - UFBA

morjanessa@gmail.com

2 Doutorado

Universidade Federal da Bahia - UFBA

emarques@ufba.br

${ }^{3}$ Doutorado

Universidade Federal da Bahia - UFBA

eduardo.albuquerque@fiocruz.br

4 Doutorado

Universidade de Taubaté - Unitau /Instituto de Estudos Avançados - IEA/USP

marcela.moraes@unitau.br

${ }^{5}$ Doutorado

Universidade Federal da Bahia - UFBA

ematosribeiro@gmail.com

${ }^{6}$ Doutorado

Universidade Federal do Vale do São Francisco Univasf

platini.fonseca@univasf.edu.br

Recebido em: 04 mar. 2019

Aprovado em: 13 fev. 2020
Resumo: Objetivo do artigo é analisar o uso de softwares sociais e de ferramentas colaborativas no processo de inovação em empresas de serviço de tecnologia. Os dados foram coletados a partir de entrevistas semiestruturadas com os gestores e os resultados apontam a contribuição destas tecnologias no apoio ao processo de inovação como um todo, intensificando a colaboração inter e intraorganizacional. Há evidências que as ferramentas se mostram eficazes e acessíveis ao público, facilitando o trabalho simultâneo e compartilhado para a geração de inovações. Destacam-se a relevância das mídias e de ferramentas da Google no apoio ao processo de busca, lançamento e aprendizagem da inovação, além do uso de ferramentas de edição simultânea e de controle de ações individuais na fase da implementação. Os dados indicam ainda a necessidade de as empresas utilizarem diversas ferramentas com propriedades diferentes e complementares para o gerenciamento do processo de inovação.

Palavras-chave: Ferramentas sociais e colaborativas. Processo de inovação.

Abstract: The purpose of this article is to analyze the use of social software and collaborative tools in the innovation process in technology service companies. The data were collected from semi-structured interviews with the managers and the results point out the contribution of these technologies in supporting the innovation process as a whole, intensifying inter and intraorganizational collaboration. There is evidence that the tools are effective and accessible to the public, facilitating simultaneous and shared work for the generation of innovations. We highlight the relevance of Google's media and tools in supporting the search process, launch and learning of innovation, and the use of simultaneous editing tools and individual actions control in the implementation phase. The data also indicate the need for companies to use various tools with different and complementary properties to manage the innovation process.

Keywords: Social and collaborative tools. innovation process. 


\section{Introdução}

A globalização e os avanços tecnológicos trouxeram um novo arranjo para a estrutura das organizações (Borges, 2008), destacando os recursos intangíveis, que se traduzem na criação, difusão e aplicação dos conhecimentos, especialmente aliados à capacidade de inovação, contribuindo para um aumento da competitividade organizacional (Quandt, 2004). Este cenário está relacionado a uma ideia de sociedade representada pelas expressões Sociedade da Informação e Sociedade do Conhecimento (Quevedo, 2007), o que remete à capacidade de gerar e compartilhar informações e conhecimentos nas organizações, permitindo desenvolver práticas úteis à promoção da inovação.

A inovação é definida pela Organização para Cooperação e Desenvolvimento Econômico [OCDE] (2005) como a implantação de um processo, método de marketing, método organizacional, bem ou serviço novo ou significativamente melhorado nas práticas de negócio, organização do local de trabalho ou relações externas, e vem cada vez mais permeando as agendas de gestão das organizações, contribuindo para a competitividade organizacional e o crescimento econômico.

Há uma compreensão de que as empresas, independentemente do porte e do setor de atuação, devem buscar inovar para obter vantagem competitiva em um mercado cada vez mais disputado. Entretanto, ainda que em um mesmo setor, há que se considerar o porte da empresa - realidades diferentes são apontadas para médias e grandes em comparação com micro e pequenas empresas (MPEs) (Nogueira \& Oliveira, 2013), embora estas últimas tenham importância destacada, especialmente no setor de serviços (World Bank Group, 2014).

As fontes de conhecimentos inerentes ao processo de geração e difusão de inovações estão dentro e fora das organizações e contam com a participação de agentes sociais e econômicos com diferentes tipos de informação e conhecimento, que são insumos elementares da inovação (Rothwell, 1992). Dessa forma, a colaboração é vista como aspecto imprescindível para a inovação nas organizações, caracterizada por um processo interativo em que duas ou mais partes trabalhem em conjunto para atingir objetivos comuns por meio do compartilhamento de conhecimento, aprendizado e construção de consenso (Teixeira et al., 2011). Os softwares sociais e as ferramentas colaborativas, portanto, adquirem grande importância, visto que possibilitam potencializar a colaboração no processo de gestão da inovação (Tidd, Bessant \& Pavitt, 2005).

Novas soluções tecnológicas vêm sendo desenvolvidas com vistas a reunir em um único ambiente virtual de trabalho ou plataforma digital diversas ferramentas e sistemas como forma de facilitar a interação e a cooperação no desenvolvimento do trabalho, a exemplo de ferramentas de busca, conexão, editoração e compartilhamento de conteúdo e mídias sociais. McAfee (2010) as denomina Plataformas Emergentes de Softwares Sociais (PESS).

Para Dames (2006), software social é qualquer tecnologia que permita a colaboração entre duas ou mais pessoas. Segundo Cardoso (2007), são ferramentas que auxiliam o processo de produção e disseminação de conhecimentos por meio da colaboração. 
Pereira, Baranauskas e Silva (2010) esclarecem que os softwares sociais e as ferramentas colaborativas são sistemas que exigem a produção de uma grande quantidade de conteúdo pelos seus usuários, permitindo às pessoas se comunicarem e mediando qualquer forma de relacionamento social. Já Ulbricht et al. (2014) complementam que estas são ferramentas que possibilitam a correspondência de informações e conhecimentos de maneira interativa, rápida e eficiente, facilitando o trabalho em grupo e a construção de projetos inovadores e criativos.

Miranda e Santos (2015) acrescentam que os softwares sociais contribuem para a gestão da inovação, enfatizando seu papel para a colaboração intraorganizacional.

A partir desse contexto, notadamente com o aumento da disponibilidade de tecnologias digitais, este trabalho teve o objetivo de analisar o uso de softwares sociais e ferramentas colaborativas no processo de inovação em empresas de serviços de tecnologia, de modo a identificar possíveis contribuições dessas tecnologias digitais em atividades desta natureza.

Para tanto, foi realizado um estudo de casos múltiplos em quatro empresas de serviços de tecnologia sediadas na cidade de Salvador, Estado da Bahia. As empresas foram escolhidas por adotarem práticas inovadoras e por fazerem uso de recursos tecnológicos como apoio à atividade de inovação são recorrentes e inerentes às suas atividades.

\section{Fundamentação teórica}

\subsection{Uma aproximação à conceituação do processo de inovação}

A gestão da inovação abrange diversos conceitos, mas todos têm por base a sistematização de processos internos da organização e convergem para o surgimento de algo novo a partir da identificação de uma oportunidade de inovação. Para Tidd, Bessant e Pavitt (2005), a inovação é um processo de fazer de uma oportunidade uma nova ideia e colocá-la em uso de maneira mais ampla possível. Já Chibás, Pantaleón e Rocha (2013) entendem a inovação como uma iniciativa modesta ou revolucionária que surge como uma novidade para a organização e para o mercado, que pode trazer resultados econômicos para a empresa se aplicadas na prática -seja a inovação ligada a tecnologia, gestão, processos ou modelo de negócios".

Para que uma empresa tenha sucesso na inovação, é necessário considerar vários subprocessos relacionados à gestão e administração da inovação (Cohn, 2013), tema recente, mas considerado relevante no contexto empresarial.

Diferentes modelos de processo de inovação podem ser identificados na literatura, dentre os quais é possível destacar o de Cooper (1993), que foca no desenvolvimento de novos produtos (NPD) sob a ótica da inovação tecnológica. Este autor defende que o NPD seja fragmentado em estágios predeterminados e com atividades já previstas para cada um deles, sendo tais atividades paralelas e interfuncionais. 
Pugh (1991) apresenta outro modelo de processo de inovação chamado Totaldesign, com um olhar voltado para o mercado e a satisfação das necessidades do usuário. Pelo fato de este modelo estar subordinado a um ator externo, é caracterizado por complexidade e incertezas técnicas.

Rozenfeld et al. (2006) trazem um processo composto por três macrofases distintas de desenvolvimento, que passam pelo planejamento estratégico dos produtos, geração de ideias que dão origem a vários processos de desenvolvimento de produtos distintos e a descontinuidade dos mesmos.

Thomas (1993) considera que o processo de inovação envolve atividades pré e pós-lançamento (programas de marketing e medição de resultados). Seu modelo parte da premissa de que cada produto é específico e vivencia diferentes dimensões no processo de NPD (ideias, conceitos, protótipos, produtos e programas de marketing), o que demanda uma dinâmica de maturidade diferente para cada dimensão, bem como maior flexibilidade no desenvolvimento.

O modelo de Roberts (1988) para o desenvolvimento de tecnologias envolve atividades e decisões que dependem da sua natureza.

A despeito das diferenças, os modelos citados apontam que o desenvolvimento das inovações segue um conjunto de estágios e decisões que iniciam com a materialização da oportunidade a partir da construção de uma ideia, seguindo para o seu desenvolvimento e finalmente para o lançamento. Neste sentido, o modelo de Tidd, Bessant e Pavitt (2005) faz menção à estrutura organizacional de apoio ao processo de inovação, sem, contudo, ser prescritivo com relação a uma possível estrutura ideal. Para os autores, o modelo é útil por focar aspectos fundamentais do desafio da gestão da inovação. Segundo esses autores, a inovação é uma atividade genérica, associada à sobrevivência e ao crescimento, e seu processo apresenta as seguintes fases:

a) Procura: envolve detectar sinais do ambiente sobre potencial de mudança, tais como novas oportunidades tecnológicas, ou necessidades de mudança impostas ao mercado. Fontes de evidências: forças internas e externas e mecanismos de detecção de oportunidades.

b) Seleção: envolve selecionar oportunidades tecnológicas e mercadológicas entre as várias disponíveis, levando em conta a estratégia utilizada pelas empresas e o ajustamento das ideias à mesma. Fontes de evidências: análise de oportunidades, estratégia utilizada, ajustamento da ideia ao negócio.

c) Implementação: transformação das ideias potenciais, após seleção, em alguma forma de realidade, a partir da aquisição de novo conhecimento, da execução do projeto e do lançamento e sustentação da inovação. Fontes de evidências: combinação de diferentes formas de conhecimento, mercado preparado para a inovação, divisão de tarefas, interação entre as atividades, foco nos consumidores, capacitação e treinamento, preparação do mercado. Vale destacar que nesta fase do processo de inovação Tidd, Bessant e Pavitt (2005) preveem 3 subfases, cada uma com seus respectivos objetivos, a saber: i) Aquisição, que visa 
a combinação de diferentes e novos conhecimentos sobre a ideia anteriormente selecionada, criando condições para uma inovação eficaz; ii) Execução, que contempla a maior utilização dos recursos disponíveis, a divisão de tarefas para execução do projeto e o comprometimento das equipes de trabalho; e iii) Lançamento/Sustentação, que foca nas necessidades dos consumidores e preparação das equipes e do mercado, bem como manutenção da inovação, se necessário modificando-a.

d) Aprendizagem: reflexão do processo de gestão da inovação como um todo, revisando etapas e registrando as lições aprendidas. Fontes de evidências: "reinovação", "Lições Aprendidas".

Vale destacar que o processo de inovação não ocorre sem interrupções, estando sujeito a uma série de influências internas e externas que moldam o seu resultado. Essas influências externas podem ser organizadas em três grupos de fatores contextuais: o contexto estratégico para a inovação, a capacidade inovadora da organização e a conexão entre a organização e elementos essenciais em seu cenário externo (Tidd, Bessant \& Pavitt, 2005) (Figura 1).

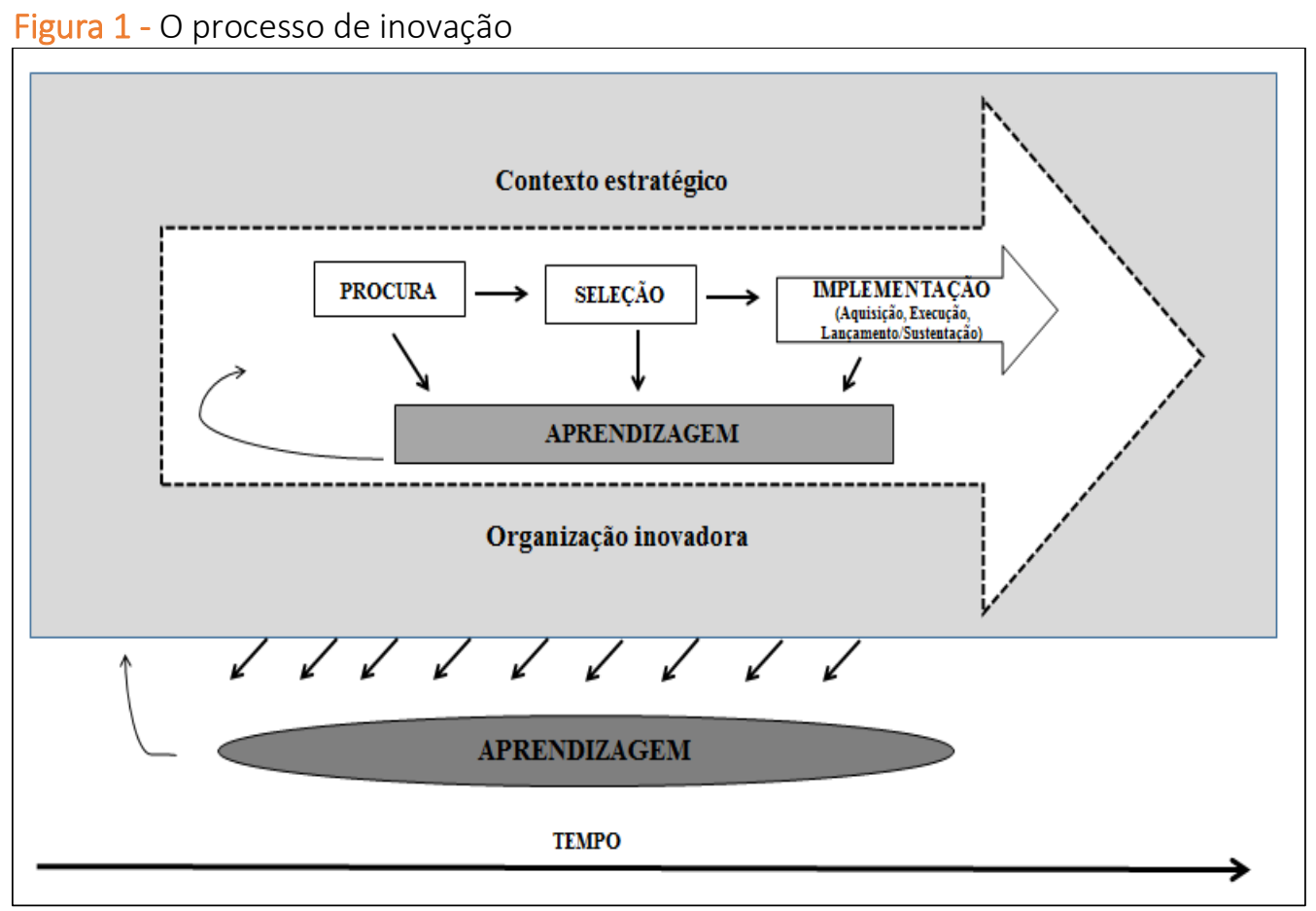

Fonte: Elaborada pelos autores, adaptada de Tidd, Bessant e Pavitt (2005).

Embora abarque decisões e ações tomadas internamente, a inovação tem uma natureza intraorganizacional, mas, por contemplar uma necessidade de compreender o ambiente e interagir com ele, tem se mostrado cada vez mais interorganizacional, e as tecnologias de informação e comunicação (TIC) exercem papel fundamental, ao aproximar pessoas, informações e conhecimentos. 


\subsection{Softwares sociais e as ferramentas colaborativas}

Com a evolução do modelo unidirecional da internet (Castells, 1999), com o aumento do interesse das organizações em exercitarem a cooperação na construção de conteúdos (Rantanen, 2005) e em mudarem cultura dentro de configurações de desenvolvimento de produtos (Rohmann, Heuschneider \& Schumann, 2015) as novas soluções tecnológicas, chamadas por McAfee (2010) de Plataformas Emergentes de Softwares Sociais (PESS), vêm sendo desenvolvidas com vistas a reunir, de forma interativa, em um único ambiente virtual de trabalho, uma plataforma digital com diversos sistemas e ferramentas de busca, editoração e compartilhamento de conteúdo.

As propriedades materiais dos Softwares Sociais tendem a facilitar diversas atividades no desenvolvimento do trabalho. McAfee (2010) destaca seis possibilidades: i) edição em grupo, que permite a colaboração na produção de um documento ou trabalho de forma centralizada, com controle de versões e edição simultânea, como wikis, Google Docs e Google Streadsheet; ii) autoração, que é a geração e disponibilização de conteúdo on-line; iii) pesquisa distribuída, também chamada broadcast search, que permite realizar consultas em diferentes fóruns públicos para aproximar demandantes e ofertantes de ideias e conhecimentos; iv) formação e manutenção de redes sociais, que permitem capturar os saberes das pessoas; v) inteligência coletiva, que trata do uso de tecnologias para gerar respostas a partir de informações dispersas; vi) auto-organização, que permite às pessoas criar e moldar comunidades e recursos ao longo do tempo de forma espontânea.

Outros autores trazem definições diferentes, porém complementares, para software social, em uma visão menos integrada. Para Dames (2006), a colaboração pode ocorrer de forma síncrona ou assíncrona, abrangendo continentes ou simplesmente um prédio de escritórios. Já Cardoso (2007) entende que software social são ferramentas que podem auxiliar o processo de produção e disseminação de conhecimento pelas empresas.

As ferramentas colaborativas auxiliam no desenvolvimento de tarefas realizadas por um grupo, o qual busca, por meio do trabalho coletivo, cumprir um projeto ou um objetivo em comum. Como exemplos é possível citar: Really simple Syndication (RSS), que permite a assinatura on-line de serviços; mash-ups, que agrega conteúdos de fontes distintas em um novo serviço; redes peer-to-peer (P2P), através das quais são compartilhados arquivos eletrônicos; podcasts e videocasts.; wikis; website; rating, serviço que permite a avaliação de diferentes itens; e tagging, que permite a classificação de informações através de palavras-chave.

Machado (2009) cita que as ferramentas Web 2.0 são atrativas, simples e úteis, e têm como características, dentre outras: a produção compartilhada, a utilização de ferramentas gratuitas para atividades particulares ou coletivas, a construção coletiva de conhecimento, a realização de serviços via web.

Camargo (2004) complementa ao trazer as principais funcionalidades dos sistemas colaborativos: coautoria; repositório de documentos; audioconferência e videoconferência; agendas; 
gerador de formulários; suporte a decisão; bate papo, chat ou messaging; fóruns de discussão; correio eletrônico; reuniões virtuais; e workflow.

Para Perez (2002), muitas empresas que têm investido na integração dos Softwares Sociais aos processos de trabalho declaram que é possível observar impactos positivos no ambiente interno e na relação com os stakeholders. De forma semelhante, observa-se que os gestores percebem um aumento significativo na colaboração e comunicação organizacional e na gestão do conhecimento com a utilização de ferramentas colaborativas na execução das tarefas nas organizações, elementos importantes para o processo de inovação, revelando um aumento constante, inclusive entre os empregados, da incorporação de Softwares Sociais na execução do trabalho (Andriole, 2010; Bughin, Chui \& Miller, 2009).

\subsection{Softwares sociais/ferramentas colaborativas no processo de inovação}

Impulsionando e sendo impulsionada pelas TIC, a internet tem se revelado fundamental para o cenário da inovação, dando vez à mudança organizacional (Tidd, Bessant \& Pavitt, 2005). Considerada uma evolução do modelo unidirecional da internet, a Web 2.0 representa um ambiente mais interativo, caracterizado pelo incentivo à colaboração e participação do usuário, contribuindo para a interação e comunicação entre as pessoas, e os movimentos e ações das organizações precisam dar a devida atenção ao potencial destas novas mídias, inclusive como suporte a processo de inovação nas organizações (Castells, 1999).

Embora o uso de ferramentas da Web 2.0 em atividades de pesquisa e desenvolvimento (P\&D) e seus benefícios não sejam completamente novos (Hinchcliffe, 2007; Piller, Vossen \& Ihl, 2012), poucos trabalhos sobre o tema foram identificados na literatura. Estudos empíricos (Bertoni, Chirumalla \& Johansson, 2012; Peltola \& Mäkinen, 2014; Tucker, Barczak \& Hultink, 2014) analisam a adoção e difusão destas tecnologias em outras práticas organizacionais e apontam que o uso de softwares sociais no processo de $P \& D$ como um todo é tímido e pontual, inclusive no que tange à compreensão do papel destas tecnologias em fases distintas do processo (Baum, Spann, Füller \& Pedit, 2013; He \& Yan, 2013; Tucker, Barczak \& Hultink, 2014). O quadro 1 relaciona as possíveis contribuições das tecnologias digitais para as fases do processo de inovação. 
Quadro 1 - Quadro relacional: processo de inovação e os softwares sociais/ferramentas colaborativas

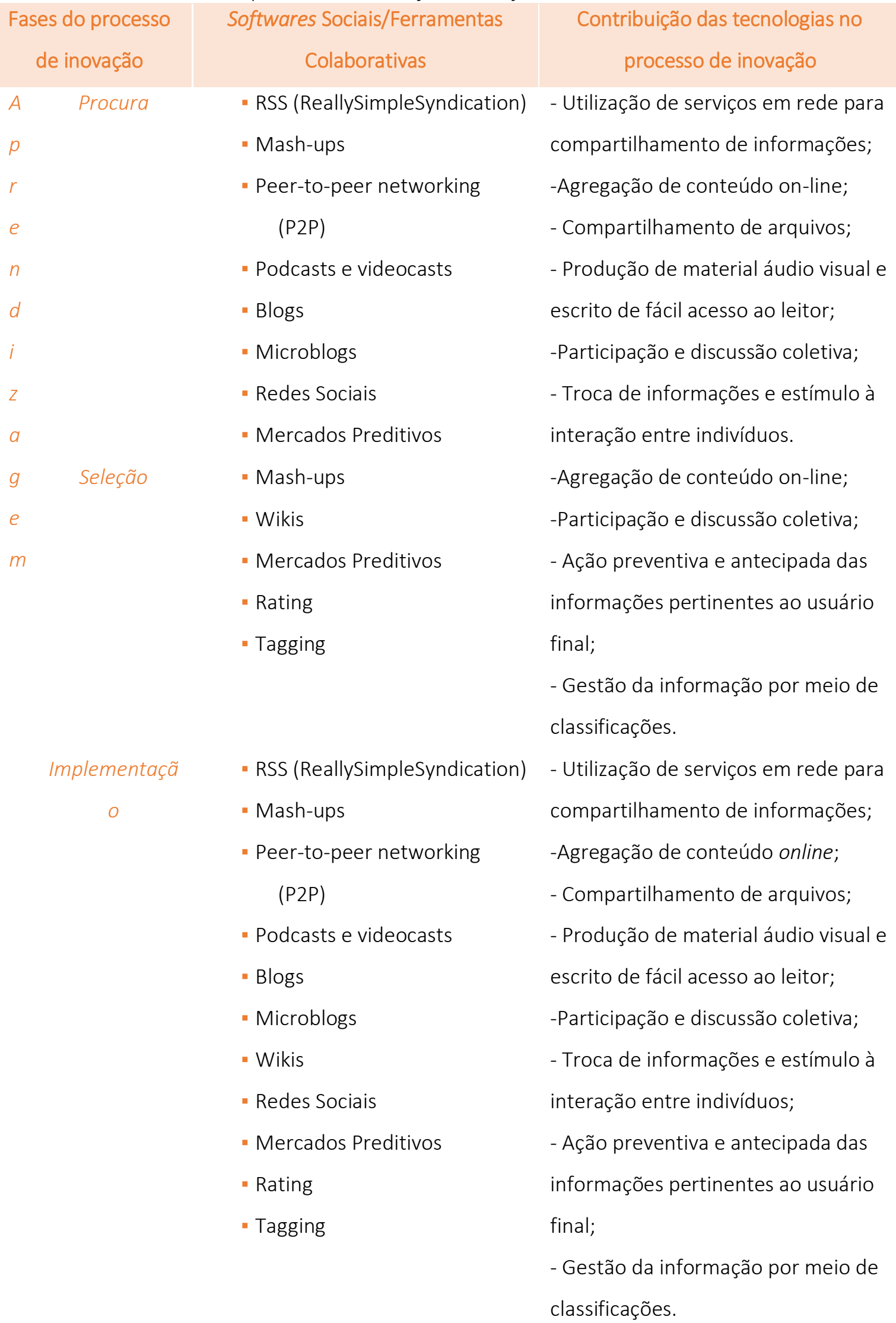

Fonte: Elaborado pelos autores a partir de Bughin e Chui e Miller (2009) e Miranda e Santos (2015).

O quadro 2 mostra como as propriedades materiais dos softwares sociais/ferramentas colaborativas interagem e contribuem com o processo de inovação. 
Quadro 2 - Quadro relacional: processo de inovação e as propriedades dos softwares sociais/ferramentas colaborativas

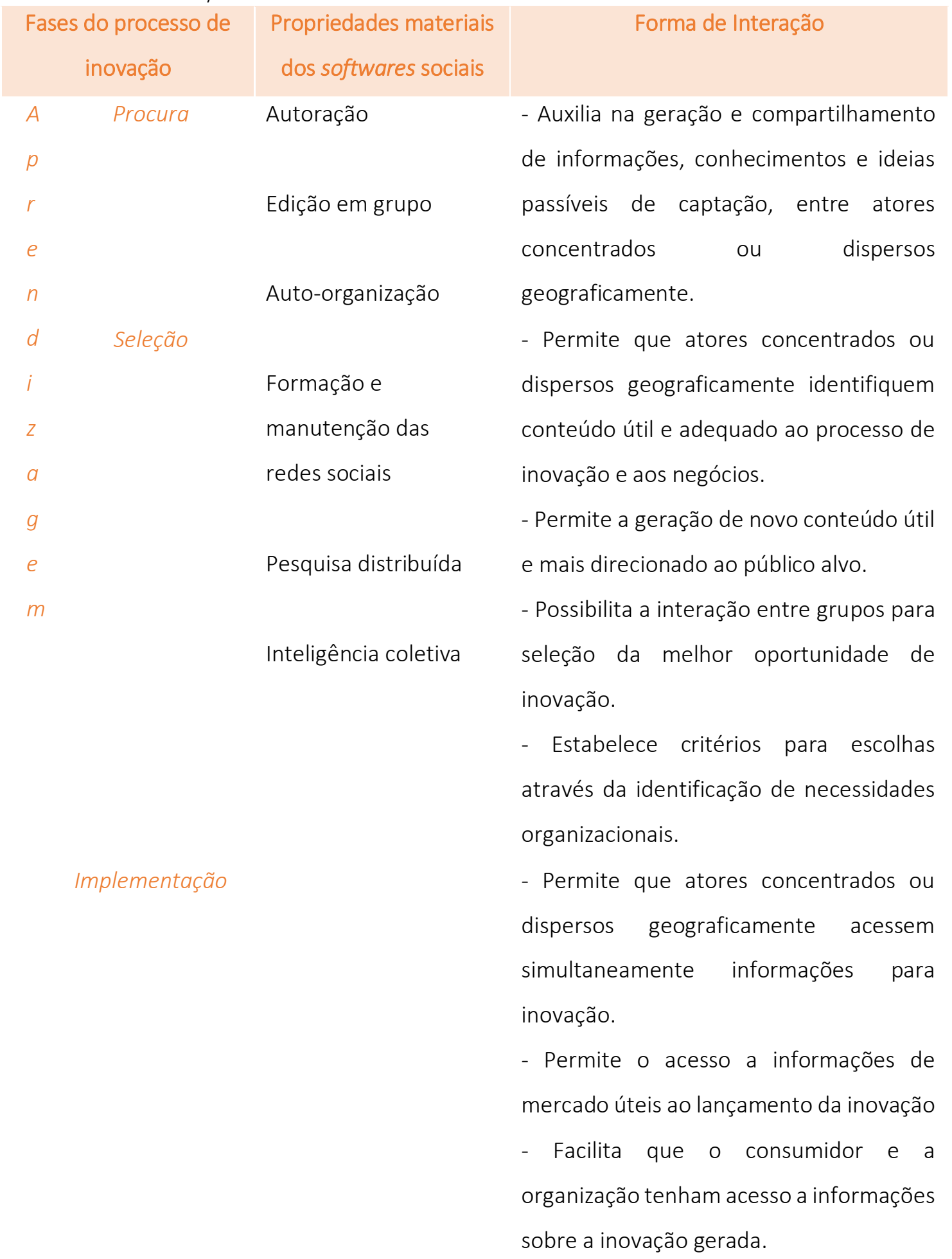

Fonte: Elaborado pelos autores a partir de Miranda e Santos (2015), West e Gallagher (2006), Jue, Marr e Kassotakis (2010), Awazu et al. (2009) e Song e Song (2010).

\section{Procedimentos metodológicos}

Os dados deste estudo de casos múltiplos foram coletados por meio de entrevistas semiestruturadas com gestores de quatro empresas de serviços de tecnologia sediadas na cidade de Salvador, na Bahia. As entrevistas foram presenciais e cada uma teve cerca de duas horas de duração. 
O roteiro das entrevistas foi construído a partir das fontes de evidências do processo de inovação descritos por Tidd, Bessant e Pavitt (2005), de forma a identificar, simultaneamente, a presença e forma de uso dos softwares sociais e das ferramentas colaborativas em cada uma das fases do processo de inovação. O quadro 3 apresenta um quadro com os indicadores identificados na literatura para cada fase do processo de inovação.

A partir dos indicadores de cada fase do processo de inovação (procura, seleção, implementação e aprendizagem), foi construído o roteiro de entrevista utilizado na pesquisa. As 24 perguntas do roteiro foram organizadas em quatro partes referentes às fases do processo de inovação com a finalidade de facilitar a transcrição e análise dos dados.

As transcrições das entrevistas de cada empresa foram lidas na íntegra e analisadas com base nos indicadores de cada fase do processo de inovação, o que possibilitou a identificação das evidências empíricas e sua associação com os construtos do quadro de análise.

Uma vez organizadas, as evidências foram analisadas a fim de identificar as tecnologias digitais e suas propriedades em cada fase do processo de inovação de cada uma das empresas selecionadas. 
Quadro 3 - Quadro de indicadores da gestão da informação - ferramentas (SS/ferramentas colaborativas) no processo de inovação

\begin{tabular}{|c|c|c|c|}
\hline Dimensão & $\begin{array}{l}\text { Fases do processo de } \\
\text { inovação }\end{array}$ & $\begin{array}{l}\text { Propriedades dos } \\
\text { SS/ferramentas } \\
\text { colaborativas }\end{array}$ & Indicadores \\
\hline \multirow{4}{*}{ Organizacional } & \multirow{4}{*}{$\begin{array}{l}\text { Procura } \\
\quad * \\
\text { Seleção } \\
\quad * \\
\text { Implementação } \\
\quad * \\
\text { Aprendizagem }\end{array}$} & $\begin{array}{l}\text { (SSa) Autoração de } \\
\text { conteúdo online }\end{array}$ & $\begin{array}{l}\text { - Geração conjunta de conteúdo online. } \\
\text { - Distribuição de atribuições. } \\
\text { - Compartilhamento de conhecimentos, competências, experiências e ideias. } \\
\text { - Atores concentrados ou dispersos geograficamente. } \\
\text { - Atuação simultânea ou não. } \\
\text { - Atuação espontânea ou não. } \\
\text { - Atuação ativa ou passiva. } \\
\text { - Reflexão de ações/Lições Aprendidas. }\end{array}$ \\
\hline & & $\begin{array}{l}\text { (SSe) Edição em grupo de } \\
\text { informações online }\end{array}$ & $\begin{array}{l}\text { - Colaboração para geração conjunta de um produto centralmente armazenado. } \\
\text { - Distribuição de atribuições. } \\
\text { - Compartilhamento de conhecimentos, competências, experiências e ideias. } \\
\text { - Atores concentrados ou dispersos geograficamente. } \\
\text { - Atuação simultânea, essencialmente em grupos restritos. } \\
\text { - Atuação espontânea ou não. } \\
\text { - Atuação ativa ou passiva. } \\
\text { - Reflexão de ações/Lições Aprendidas. }\end{array}$ \\
\hline & & $\begin{array}{l}\text { (SSao) Auto-organização de } \\
\text { informações online }\end{array}$ & $\begin{array}{l}\text { - Criação de comunidades e recursos valiosos, espontâneos e evolucionários. } \\
\text { - Compartilhamento de conhecimentos, competências, experiências e ideias. } \\
\text { - Atores concentrados ou dispersos geograficamente. } \\
\text { - Atuação simultânea ou não. } \\
\text { - Atuação espontânea. } \\
\text { - Atuação ativa. } \\
\text { - Reflexão de ações/Lições Aprendidas. }\end{array}$ \\
\hline & & $\begin{array}{c}\text { (SSrs) Formação e } \\
\text { manutenção das redes } \\
\text { sociais }\end{array}$ & $\begin{array}{l}\text { - Captura de saberes } \\
\text { - } \text { - Atompartilhamento de conhecimentos, competências, experiências e ideias. } \\
\text { - Atuação simultânea ou dispersos geograficamente. } \\
\text { - Atuação espontânea. } \\
\text { - Atuação ativa ou passiva. } \\
\text { - Reflexão de ações/Lições Aprendidas. }\end{array}$ \\
\hline
\end{tabular}


- Divulgação de problemas para os quais necessita de ajuda.

- Distribuição de atribuições.

- Compartilhamento de conhecimentos, competências, experiências e ideias.

(SSp) Pesquisa distribuída de informações online

(SSic) Inteligência coletiva (saber das multidões)
- Atores concentrados ou dispersos geograficamente.

- Atuação simultânea.

- Atuação espontânea ou não.

- Atuação ativa ou passiva.

- Reflexão de ações/Lições Aprendidas.

- Geração de respostas a partir de um grupo disperso.

- Distribuição de atribuições.

- Compartilhamento de conhecimentos, competências, experiências e ideias.

Atores concentrados ou dispersos geograficamente.

- Atuação simultânea.

- Atuação espontânea ou não.

- Atuação ativa ou passiva.

- Reflexão de ações/Lições Aprendidas.

Fonte: Elaborado pelos autores (2016) a partir de Miranda e Santos (2015), West e Gallagher (2006), Jue, Marr e Kassotakis (2010), Awazu et. al. (2009) e Song e Song (2010). 


\section{Apresentação e análise dos resultados}

\subsection{As empresas de tecnologia estudadas}

As empresas que participaram do estudo atuam no mercado de TIC na Bahia. A ADSS Presença Digital foi fundada em 2010 e tem seis colaboradores, funcionando em uma estrutura de coworking. Seu foco de atuação é o marketing digital, desenvolvendo em paralelo projetos inovadores de cunho tecnológico, voltados especialmente para a construção de sites e aplicativos.

A INOVIT Consultoria e Soluções em TI foi fundada em 1995 e conta com 19 colaboradores. Seu foco de atuação é a oferta de serviços e soluções na área de TIC, como suporte a servidores de rede e internet, implantação de políticas de segurança e monitoramento de servidores.

A MAQHIN Soluções Tecnológicas foi criada em 2009 e tem 16 colaboradores. Ela atua no desenvolvimento de soluções de TIC para pessoas e empresas, desenvolvendo sistemas sob medida, criação e gestão de aplicativos e websites, design gráfico e de comunicação, comércio eletrônico, gestão de redes sociais e negócios digitais.

A IPQ Tecnologia, com mais de 15 anos de existência e cerca de 11 colaboradores, atua no mercado de sistemas de informação, infraestrutura, redes, telecomunicações e mobilidade, tendo como principal cliente o setor de construção civil.

\subsection{Contribuição dos softwares sociais/ferramentas colaborativas para o processo de inovação}

\subsubsection{A fase da procura}

Os dados mostram que as empresas pesquisadas são constantemente incitadas a buscarem oportunidades de inovação. A MAQHIN cria novos aplicativos ainda não lançados no mercado, ao mesmo tempo em que busca responder a demandas dos clientes. A ADSS, por sua vez, busca repensar o próprio modelo de negócio, enquanto a INOVIT busca melhorar seus processos internos.

Essas oportunidades são identificadas pelas empresas através de ações presenciais, como reuniões com atores internos e externos para captação de ideias, o preenchimento de formulários específicos para captação informações relevantes para o processo de inovação, como os Canvas da Inovação, e a participação em feiras, eventos e cursos. Os entrevistados relataram também o uso de softwares sociais e ferramentas colaborativas para fins de inovação, a exemplo de bancos de dados virtuais de ideias para captação, votação e seleção de oportunidades de inovação - na empresa MAQHIN, é utilizado a Máquina de Inovação, enquanto INOVIT e IPQ utilizam o Banco de Ideias do software SisJOIN de apoio à gestão da inovação. Na IPQ foi identificada ainda uma tentativa frustrada de utilização de uma plataforma colaborativa chamada NEON, que apresenta funcionalidades como chat, grupos de discussão e biblioteca, mas que foi posta de lado devido ao uso de outras soluções que apresentam funcionalidades semelhantes, como os softwares Gtalk e Skype, e fóruns de discussão e repositórios de dados criados e mantidos pela empresa. A empresa ADSS utiliza também os softwares SM2, Hootsuit e Sprout Social, que são voltados para o atuação e gerenciamento da presença das empresas em mídias sociais. 
Esses softwares foram apontados pelos entrevistados como soluções que contribuíram para a busca por oportunidades de inovação, pois tornaram mais fácil e eficiente a presença das empresas nas mídias sociais e as aproximou dos clientes e das principais fontes de ideias. O uso dessas soluções tecnológicas em paralelo confirma o que argumentou Rantanen (2005) quanto ao uso integrado de diferentes soluções tecnológicas para promover a cooperação. O fato de mais de uma empresa utilizar ferramentas semelhantes e complementares corrobora com McAfee (2010) quanto à criação de uma plataforma digital composta por diferentes sistemas e ferramentas.

A ferramenta Google Trends, que mostra, por meio de gráficos de frequência, os termos mais buscados em um passado recente, também foi considerada útil na busca por tendências e oportunidades de inovação, como apontou o entrevistado da ADSS. O Trello, um "kanban" virtual de acompanhamento de atividades simultâneas, também foi citado na entrevista com a ADSS como uma ferramenta colaborativa usada no gerenciamento do processo inovativo para acompanhamento da identificação e definição do foco nas ideias coletadas pela empresa. O uso de ferramentas de editoração e compartilhamento de conteúdo foi previsto por McAfee (2010) e Cardoso (2007), entre outros.

Como exemplo de interação com o ambiente externo, as empresas são procuradas por outros atores para desenvolvimento de projetos inovadores, permitindo a identificação de nichos, problemas e oportunidades advindas dos ambientes interno e externo. O Researchgate é uma plataforma colaborativa que concentra uma série de pesquisadores e suas produções científicas em rede, considerada útil para a ADSS por permitir acesso a referências de trabalhos científicos voltados ao desenvolvimento de plataformas tecnológicas, uma necessidade de um dos seus projetos, o que reflete a importância da interação entre o conhecimento técnico e o conhecimento científico para geração de inovações. O Github, um repositório com funcionalidades de rede social voltado para desenvolvedores de software, também foi citado nas entrevistas com participantes da ADSS e da MAQHIN como uma ferramenta que possibilita que seus projetos de novos produtos sejam desenvolvidos mais rapidamente.

As entrevistas mostraram também que a empresa IPQ busca oportunidades de inovação e ideias para demandas existentes no Google Alerts e Google Notícias, além do microblog Twitter. As mídias sociais Facebook e Linkedln também foram citados pelos entrevistados como ferramentas comumente usadas na fase da procura por oportunidades de inovação. Segundo os entrevistados, essas ferramentas são utilizadas para captar e trazer para a organização ideias que estão sendo atualmente discutidas no ambiente externo, especialmente por atuais e potenciais clientes.

As quatro empresas possuem grupos no aplicativo para smartphones WhatsApp para discussão de assuntos relativos a inovação, mas os entrevistados da ADSS e MAQUIN relataram que a ferramenta que mais utilizam hoje para essa finalidade é o Slack, uma solução para trabalho em equipe que permite a criação de comunidades para compartilhamento de interesses comuns. 
Para colaboração em atividades realizadas remotamente, a ADSS utiliza o Google Docs, que permite o trabalho colaborativo inter e intraorganizacional, inclusive para elaboração e gestão de contratos de inovação. O Google Form, um gerador e gerenciador de formulários online integrado ao Google Docs, também foi apontado como uma ferramenta utilizada por esta empresa em projetos de inovação, nos quais pode haver participação de todos os seus colaboradores. Os benefícios da integração dessas ferramentas aos processos de trabalho, inclusive para interação com outras partes interessadas, como abordara Perez (2002) e Andriole (2010), justifica sua utilização na empresa.

As possibilidades decorrentes do uso de ferramentas Web 2.0 foram apontadas por Machado (2009) e Camargo (2004), e foram identificadas nas quatro empresas, mas os entrevistados relataram também obstáculos que se apresentam em todas as fases de gestão da inovação, dentre os quais se destacam a dificuldade de colaboração intra e especialmente interorganizacional para geração de inovação, como apontaram os entrevistados da ADSS, e a necessidade de disciplina para realizar a busca por informações relevantes e de qualidade, relatada nas entrevistas com os informantes da INOVIT, a operacionalização das ideias captadas, que se acumulam, segundo os entrevistados da MAQHIN, e, na IPQ, o estímulo para geração de ideias nos colaboradores de todos os níveis da organização.

A motivação para buscar oportunidades de inovação também é um fator de destaque quando se trata de barreiras à inovação, visto que a vontade inovar e os possíveis benefícios da inovação favorecem essa busca. Neste sentido, as quatro empresas construíram Políticas de Inovação que preveem, dentre outros aspectos, a premiação dos colaboradores envolvidos na inovação. Para aferir o desempenho dos colaboradores, as empresas utilizam planilhas no Google Drive ou uma funcionalidade do sistema SisJOIN, no caso da INOVIT.

Nos casos estudados, a procura, que é anterior à implementação da inovação (Thomas, 1993), utiliza diferentes ferramentas sociais para detectar no ambiente oportunidades, como preveem Tidd, Bessant e Pavitt (2005) e Khurana e Rosenthal (1998).

\subsubsection{A fase da seleção}

Para selecionar e analisar as oportunidades de inovação identificadas, alguns elementos são analisados pelas empresas, como os recursos humanos disponíveis na organização, a sua capacidade de produção e o escopo dos seus projetos. O processo de seleção dos projetos tem por base sua viabilidade, avaliada nas empresas ADSS e MAQHIN quanto às dificuldades que apresentam e sua relevância.

A IPQ Tecnologia utiliza as mídias sociais para a busca de novas informações para seleção das oportunidades de inovação identificadas na fase anterior, o que conta com a participação de todos os seus colaboradores, que podem colaborar através do Banco de Ideias do sistema SisJOIN. 
O Trello e o Google Drive foram também utilizados pelas empresas para compilar informações pertinentes aos seus projetos, de modo que todos os envolvidos pudessem ter acesso às informações. Ao selecionar as oportunidades de inovação, as empresas ajustam as ideias à estratégia organizacional, com um conhecimento inicial do mercado no qual estão inseridas. Neste processo, as empresas utilizam diferentes plataformas tecnológicas e ambientes virtuais, como o Nielsen, que fornece relatórios de uso da internet, a start up Hot Content, agência de relacionamentos que busca aproximar as marcas de seus consumidores através da divulgação da marca, e o Portal Resultados Digitais, que envia relatórios mensais sobre hábitos de consumo. A ADSS e a MAQHIN também realizam nesta fase uma coleta de dados por meio de entrevistas em campo, usando, para isso, um formulário no Google Forms, o que ajudou no alinhamento da inovação à estratégia da empresa, na medida em que permitiu conhecer melhor os hábitos e preferências de seus potenciais consumidores.

Postagens e feedbacks realizados pela ADSS, bem como clientes em redes e mídias sociais tais como YouTube, Facebook, Twitter, Instagram e blogs também ajudam a empresa a repensar e remodelar seus projetos de inovação, selecionando, a partir dos comentários, as melhores possibilidades de inovação alinhadas à organização.

Essas ferramentas relatadas pelos entrevistados cobrem uma parte do que foi apresentado por Camargo (2004) como funcionalidades importantes dos sistemas colaborativos. Em maior ou menor escala, as empresas interagiram com atores externos pertinentes aos seus projetos de inovação, como clientes, fornecedores e outras organizações, e as mídias sociais são percebidas pelos entrevistados como úteis e eficazes para estreitar essas relações. Divulgações pelo WhatsApp também são muito úteis e amplamente utilizadas nesta fase para apresentar, de forma simples e rápida, o desenvolvimento preliminar de uma inovação, possibilitando a seleção e o aperfeiçoamento das caraterísticas do novo produto ou serviço.

As ideias não utilizadas inicialmente pelas empresas a partir da fase da seleção também são registradas no Google Drive em pastas virtuais setorizadas. Periodicamente as empresas revisitam tais ideias, utilizando, no caso da ADSS, lembretes enviados pelo Google Calendar, para reavaliação da possibilidade de implementação das mesmas.

O uso dessas ferramentas permite que decisões relacionadas à sua estratégia de inovação sejam tomadas com segurança, como previsto por Tidd, Bessant Pavitt (2005).

\subsubsection{A fase da implementação}


A fase da Implementação se inicia pela Subfase da Aquisição, que prevê a combinação de conhecimento interno e adquirido externamente para oferecer uma solução mais detalhada para a lacuna identificada na fase da Procura, no processo de inovação.

Na obtenção dos novos conhecimentos, a divisão adequada de tarefas e a integração das atividades da empresa para a execução do projeto mostram-se relevantes.

As empresas utilizam principalmente o Google Trends, que permite a busca de temas referentes à inovação para aprofundamento da ideia/temática inicialmente selecionada, por meio de palavraschaves ou expressões. Além de portais de notícias, mídias sociais, websites, como Twitter também são fontes de informações pertinentes a essas atividades, que permitem a produção e disseminação de conhecimentos, no mesmo sentido que Cardoso (2007) apontou, e são gratuitas e utilizadas para formação de comunidades virtuais de pessoas que compartilham interesses, como Machado (2009) argumentou, reforçando uma das possibilidades previstas por McAfee (2010).

O uso do Google Docs em geral é constante nesta fase, nas empresas pesquisadas, visto que, por se tratar de uma fase de aquisição de novas informações que complementem e aperfeiçoem a ideia inicial, necessita de registro, compartilhamento e ações simultâneas entre todos os envolvidos. De acordo com McAfee (2010), esta seria uma ferramenta de edição em grupo, que permite que a produção do conhecimento nas empresas seja realizada com a colaboração de diversas pessoas.

Para esta etapa a MAQHIN utiliza o Gitlab, um gerenciador de repositório de software, para armazenar o histórico das perguntas e dúvidas eventualmente feitas por outros colaboradores, o que pode acontecer de forma assíncrona, como previu Dames (2006).

Como obstáculos à inovação neste estágio as empresas citam os fatores tempo e disciplina das partes envolvidas no desenvolvimento dos projetos, bem como o acesso a informações de qualidade, sendo considerados pontos críticos de sucesso. Para os gestores é um desafio gerir atividades de inovação que ocorrem simultaneamente, com um fluxo intenso e em paralelo às outras atividades organizacionais do dia-dia. Além disso, a disciplina para sempre buscar gerar e compartilhar novas informações referentes às ideias inicialmente selecionadas é algo importante e ao mesmo tempo dinâmico para os colaboradores. Este alinhamento entre tempo e disciplina utiliza como ferramenta tecnológica de apoio o Trello.

Este estágio do processo prevê também a criação de condições que contribuam para o desenvolvimento mais direcionado da inovação. Neste sentido, os entrevistados entendem que o trabalho de divulgação é muito importante, bem como o estímulo a uma cultura de inovação na organização que seja incorporada por toda a empresa. A utilização de softwares sociais nas atividades cotidianas da organização pode levar a mudanças positivas e, consequentemente, suplantar dificuldades, como argumentou Perez (2002). 
Ainda na Subfase da Aquisição de novas informações durante a implementação dos projetos de inovação, um dos gestores entrevistados (da IPQ) vê um viés muito grande na comunicação em mídias sociais, ou por meio das próprias plataformas de distribuição de um aplicativo como forma de converter facilmente o conhecimento em serviço em interesse de comercialização pelo mesmo. Ainda, em uma relação entre empresas, o gestor cita que a participação em fóruns e comunidades de interesse é um ótimo espaço para criação do interesse pela inovação. Hoje eles apenas utilizam o fórum internamente, para discussão de ideias e do valor de uma possível inovação.

Segundo os entrevistados, este estágio apresenta como obstáculos à inovação o tempo e a disciplina das partes envolvidas nos projetos de inovação, bem como o acesso a informações de qualidade, sendo considerados pontos críticos de sucesso.

Após a aquisição de novos conhecimentos para a implantação da inovação, o processo proposto por Tidd, Bessant e Pavitt (2005) prevê a Subfase da Execução, de fato, do projeto de inovação. Neste estágio do processo, as empresas ADSS e MAQHIN utilizam o Twitter e outras mídias sociais, como o Facebook, como indicadores de preparo do mercado para a inovação. Os entrevistados afirmam que tais ferramentas colocam a empresa em contato com pessoas que se interessam pelo assunto em pauta para inovação, reforçando o entendimento de Piller, Vossen e Ihl (2012), McAfee (2010), Andriole (2010) e outros autores.

A pesquisa mostrou que as empresas utilizam diferentes ferramentas colaborativas de apoio para a execução dos projetos, como Google Docs, Google Drive, WhatsApp, Trello, Sleck (ferramenta de comunicação interna e fórum de discussão que permite debates em equipe e sobre assuntos específicos), Azana (ferramenta para divisão de tarefas que considera as habilidades individuais) e Gitlab. Essas são ferramentas que atendem às possibilidades que McAfee (2010) apontou, além de oferecer boa parte das funcionalidades apresentadas por Camargo (2004).

Para a integração das atividades a IPQ utiliza, ainda, a Metodologia Scrum, um processo padronizado que prevê uma série de etapas sequenciais consideradas necessárias para se chegar a um produto de alta qualidade no prazo e orçamento previstos, utilizando para isso o software Ice Scrum.

Os entrevistados destacaram o papel do Google Docs para acompanhamento e rápida identificação de erros durante a execução do projeto, por ter uma funcionalidade que sinaliza quem editou os documentos.

Neste ínterim, na percepção dos gestores, é um desafio gerir atividades de inovação que ocorrem simultaneamente, com um fluxo intenso e em paralelo às outras atividades organizacionais. Além disso, a busca e o compartilhamento de novas ideias exigem uma disciplina e um dinamismo por parte dos colaboradores, que podem ter o Trello como ferramenta de apoio. 
Há atividades inerentes à execução de alguns projetos de inovação que necessitam de informações atualizadas e em tempo real, e ferramentas tecnológicas como o Instagram e o Google Earth são amplamente utilizadas pelas empresas, gerando economia de custo na medida em que evitam certos deslocamentos e otimizam o tempo de elaboração do projeto de inovação.

Para a IPQ outro obstáculo além do tempo é a disciplina. Segundo o gestor é difícil gerir atividades de inovação que ocorrem simultaneamente, com um fluxo intenso e em paralelo às outras atividades organizacionais do dia-dia. Além disso, a disciplina para sempre buscar gerar e compartilhar novas ideias é algo importante e ao mesmo tempo complexo para os colaboradores. Este alinhamento entre tempo e disciplina utiliza como ferramenta tecnológica de apoio o Trello, um "kanban" virtual de acompanhamento de atividades simultâneas, inclusive.

Tendo o projeto sido executado, a inovação se prepara para a Subfase do Lançamento/ Sustentação. Nesta etapa a coleta de novas informações para a solução de problemas com foco nas necessidades dos consumidores é crucial, visto que é ele quem terá de se interessar pela mesma.

Na MAQHIN, o lançamento da inovação é caro e tem certa complexidade, pois prescinde de muitas parcerias. Neste sentido, a empresa realiza muitos planejamentos nessa área, especialmente porque ferramentas comumente usadas e mais acessíveis, como Facebook e o ecossistema da Google, já não causam impacto tão forte nesta fase do processo de inovação, resultado que contradiz Hinchcliffe (2007) e Piller, Vossen e Ihl (2012).

Já os outros entrevistados ainda entendem que as mídias sociais oferecem indicadores úteis para o lançamento de inovações, pois possibilitam à organização apresentar, inclusive, novos produtos ou serviços, de modo que o público consumidor estreite a sua relação com ele e com a empresa.

Ainda no lançamento da inovação, os entrevistados da ADSS e INOVIT veem um viés muito grande na comunicação em mídias sociais, ou através das próprias plataformas de distribuição de um aplicativo como forma de converter facilmente o conhecimento em serviço em interesse pelo mesmo. Ainda, em uma relação empresa-empresa, dois gestores entrevistados citam que a participação em fóruns e comunidades de interesse seria um ótimo espaço para criação de valor para o usuário final, embora os fóruns sejam, em geral, usados apenas internamente, para discussão de ideias e do valor de uma possível inovação. Pequenos vídeos também são criados pela ADSS para lançamento preliminar da inovação no mercado.

Como possível obstáculo nesta etapa, os entrevistados citam a dificuldade na absorção do produto, julgando ser importante aqui a divulgação do produto ou serviço no mercado, coletando informações por meio de redes sociais e Twitter, por exemplo, de forma antecipada, o que gera, ao mesmo tempo, redução de custo para a organização.

As entrevistas evidenciaram a transformação das ideias potenciais em novos produtos ou serviços nas empresas, o que envolveu a combinação de diferentes formas de conhecimento, 
tecnologias e a interação com o mercado, como argumentaram Tidd, Bessant e Pavitt (2005), Hinchcliffe (2007) e Piller, Vossen e Ihl (2012).

\subsubsection{A fase da aprendizagem}

Todos os entrevistados percebem o sucesso das inovações no mercado (um dos parâmetros que representa a aprendizagem no processo de inovação) a partir de indicadores numéricos, a exemplo da quantidade de usuários dos seus produtos e serviços. Tal pesquisa se dá também de forma presencial e virtual, junto aos clientes. Na pesquisa virtual ferramentas como o Google Docs e a rede social Facebook funcionam como bons indicadores de análise com os clientes. No caso de inovações incrementais a serem aplicadas, a ADSS cria no Google Docs um questionário com perguntas sobre a inovação, com resultados tabulados e compartilhados entre os colaboradores da organização. Esses resultados apontam para uma importância do uso de repositórios de documentos como ferramentas colaborativas para inovação, que é uma das funcionalidades apresentadas por Camargo (2004). Fórum de discussões é também uma funcionalidade apresentada pelo autor, que pode ser suprida pelas comunidades existentes no Facebook, e que é uma característica apontada por Machado (2009).

Outras ferramentas são também utilizadas para compartilhamento do conhecimento adquirido, como o sistema SisJOIN, que tem um fórum de discussões, bem como as estruturas de arquivos das redes de computadores das empresas, que são usadas como repositórios de informações e documentos. Outras ferramentas também servem a esse fim, como o Google Docs, o Dropbox e o Google Drive, que, diferentemente das redes locais de computadores, permitem o compartilhamento fora das empresas. Tais compartilhamentos representam, sobremaneira, a capacidade das empresas de aprender durante as atividades de inovação por elas fomentadas.

As quatro empresas mantêm grupos no aplicativo WhatsApp para discussões de assuntos diversos, inclusive relacionados à inovação. Nesta fase, as atividades de inovação da ADSS são monitoradas e avaliadas tendo como suporte o Google Calendar, que compartilha para todos os membros da organização um calendário com as atividades de inovação a serem realizadas ou em andamento. O Trello também cumpre tais funções junto às empresas, possibilitando, por meio do acompanhamento das tarefas, a otimização das atividades de inovação. Em ambos os casos, trata-se de uma funcionalidade prevista por Camargo (2004).

Os entrevistados afirmam que percebem o sucesso da inovação no mercado a partir do uso de algumas ferramentas tecnológicas, como o Google Analytics, que permite a avaliação do retorno sobre os investimentos em publicidade online, bem como o acompanhamento de websites, aplicativos em Flash, vídeos e mídias sociais, e o acompanhamento de quais partes de websites ou aplicativos são mais utilizados ou acessados pelos usuários através da ferramenta Hotjar. 
As mídias sociais, por meio da verificação de "curtidas", compartilhamentos, comentários e relatórios de gerenciamento, também permitem medir o sucesso da inovação no mercado, ainda que de forma experimental. Ferramentas como Facebook, Twitter e Tumblr, que permitem a publicação de textos, imagens, vídeos, links, citações e áudios, se apresentam como interfaces de análise usadas no processo de aprendizagem da inovação.

Como possíveis obstáculos nesta fase, os entrevistados apontam para a disciplina e a gestão do tempo como elementos essenciais ao sucesso da atividade. Neste sentido, além do indispensável papel dos gestores no estímulo à geração e disseminação de conhecimentos, algumas ferramentas como o Trello e o Google Calendar se destacam. O registro e a medição dos pontos fortes e fracos decorrentes das atividades de inovação também são considerados como obstáculos pelas empresas INOVIT e MAQHIN, na medida em que ainda sentem dificuldade em armazenar informações para memória organizacional.

É importante refletir aqui que o processo de inovação mapeado, embora tenha levado ao aprendizado das empresas em diferentes proporções, precisa ser mais formalizado, de modo que possa ser explorado e otimizado em outros contextos.

\section{Considerações finais}

Este estudo reforça a importância do desenvolvimento de novas e mais aprofundadas pesquisas sobre o uso dos softwares sociais e ferramentas colaborativas para a execução do processo de inovação nas empresas.

A pesquisa mostrou, ainda, que, embora essas tecnologias possuam características particulares, possibilitaram o uso simultâneo em todas as fases de gestão da inovação considerando aqui as etapas utilizadas nesta pesquisa, tomando como referência a metodologia proposta por Tidd, Bessant e Pavitt (2005). Pode-se inferir, pois, que esses recursos ao mesmo tempo em que auxiliaram a fase da procura, ajudaram também a fase da seleção visto que possibilitou realizar a busca de novas informações para uma identificação mais precisa das oportunidades de inovação, ao tempo em que contribuiu também na fase da implantação ao dotar a equipe de gestores de indicadores para o preparo do mercado para a inovação, assim como estimulando ações de aprendizagens.

Articulando os achados da pesquisa com a concepção defendida por Dames (2006), é possível afirmar que o objetivo final dos softwares sociais é a construção de uma comunidade de prática ou formação de uma rede de conhecimento onde os participantes podem dar e receber informaç̧̃es úteis, constantemente, não importando qual a distância ou forma de colaboração. Nesse sentido, os estudos de caso revelaram que a utilização intensiva de diversas ferramentas colaborativas como apoio à 
atividade de inovação ganha relevância também pelo fácil acesso, manuseio e gratuidade, o que viabiliza, assim, a ampla apropriação, incorporação e, eventualmente, desapego destes pelas empresas.

Um aspecto analisado na pesquisa, em cada fase do processo de inovação, são os obstáculos, seja de ordem subjetiva ou objetiva. Neste sentido, destacam-se: dificuldade na colaboração interorganizacional (motivação); disciplina para a busca por informações relevantes e de qualidade; operacionalização das ideias captadas; gestão do tempo pelas partes envolvidas neste processo; gestão simultânea de atividades de inovação, inclusive entre atores dispersos geograficamente; disciplina para sempre buscar gerar e compartilhar novas ideias; dificuldade na absorção do produto pelo mercado; e o registro e a medição dos pontos fortes e fracos decorrentes das atividades de inovação.

Para fins de proposições para a superação dos obstáculos diagnosticados, considera-se que a contribuição das ferramentas citadas pode materializar-se por meio das seguintes ações: uso de templates virtuais de inovação e bancos de ideias, físicos ou virtuais, para busca por oportunidades de inovação; ferramentas de gerenciamento das mídias sociais, para captação de informações atinentes às questões de inovação da empresa; "kanbans" virtuais, para acompanhamento de atividades simultâneas; plataformas colaborativas, para interação e troca de conhecimentos entre atores internos e externos à organização; ferramentas de edição e acompanhamento simultâneo, como o Google Docs e Google Drive, para atividades realizadas de forma integrada e remota; "gamificações" virtuais para motivação das partes envolvidas no processo de inovação; ferramentas para divisão de tarefas em projetos de inovação; e ferramentas com interfaces de análise interessantes usadas no processo de aprendizagem da inovação.

Identifica-se, portanto, a tendência do uso de softwares sociais e ferramentas colaborativas em empresas de serviços de tecnologia, refletindo, no entanto, para o pouco aproveitamento dessas ferramentas para a colaboração interorganizacional, ação que exige uma cultura organizacional de inovação mais fortalecida, traduzindo-se, neste sentido, em maior capacidade relacional e de empatia com atores internos e externos à organização.

Embora se reconheça que as ferramentas tecnológicas, inclusive as colaborativas, não devam prevalecer sobre as pessoas ou comunidades envolvidas em um projeto de inovação, é perceptível que as questões atinentes a tais tecnologias perpassam e prescindem de elementos como qualidade e distribuição das informações, tempo oportuno, e a própria disponibilidade e acesso às mesmas. (Dames, 2006, como citado em Miranda \& Santos, 2015).

Como limitação do estudo é importante destacar a ausência de um conjunto de dados mais amplo que permitisse um maior aprofundamento da pesquisa. Entretanto, a própria literatura sobre o tema tem destacado a baixa sistematização no processo de inovação nas empresas, o que exige investimentos de novos estudos que contribuam para ampliar a discussão sobre esse fenômeno que 
ganha, cada vez mais, relevância devido justamente às profundas e aceleradas mudanças que tem ocorrido nos padrões de gestão das organizações tendo os processos de inovação como destaque. Nesse sentido, sugere-se como agenda de estudos futuros investigar em outros setores e/ou áreas de atuação de empresas quais as contribuições trazidas pelos softwares sociais aplicados aos modelos de gestão da inovação dos processos e dos negócios.

\section{Referências}

Andriole, S. J. (2010). Business impact of Web 2.0 technologies. Communications of the ACM, 53(12), 67-79. DOI: http://doi.acm.org/10.1145/1859204.1859225.

Awazu, Y., Baloh, P., Desouza, K. C., Wecht, C. H., Kim, J., \& Jha, S. (2009). Information-communication technologies open up innovation. Research Technology Management, 52(1), 51-58. DOI: https://doi.org/10.1080/08956308.2009.11657548.

Baum, D., Spann, M., Füller, J., \& Pedit, T. (2013). Social media campains for new product introductions. Proceedings of the European Conference on Information Systems, Utrecht, Holanda, 21.

Bertoni, M., Chirumalla, K., \& Johansson, C. (2012). Social technologies for cross-functional product development: SWOT analysis and implications. Proceedings of the Hawaii International Conference on System Sciences, Maui, HI, USA, 45, 3918-3927.

Borges, M. A. G. (2008). A informação e o conhecimento como insumo ao processo de desenvolvimento. Revista Ibero-americana de Ciência da Informação,1(1), 75-196. DOI: https://doi.org/10.26512/rici.v1.n2.2008.1249.

Bughin, J., Chui, M., \& Miller, A. (2009). How companies are benefiting from Web 2.0: McKinsey Global Survey results. McKinsey Quarterly. DOI: https://www.mckinsey.com/business-functions/mckinseydigital/our-insights/how-companies-are-benefiting-from-web-20-mckinsey-global-survey-results\#.

Camargo, A. A. B. (2004). Gestão Colaborativa - Metrô de São Paulo. São Paulo, SP - Dias 26/11/2004, 02 e 03/12/2004.

Cardoso, M. H. (2007). Software social no ambiente corporativo: transformando a produção e disseminação de conhecimento nas organizações. DataGramaZero, 8(4).

Castells, M. (1999). A sociedade em rede. São Paulo: Paz e Terra.

Chibás, F. O., Pantaleon, E., \& Rocha, T. (2013). Gestão da inovação e da criatividade hoje: apontes e reflexões. Holos, 3, 15-26. DOI: http://dx.doi.org/10.15628/holos.2013.1082.

Cohn, S. (2013). A firm-level innovation management framework and assessment tool for increasing competitiveness. Technology Innovation Management Review, 3(10), 6-15. DOI: http://10.22215/TIMREVIEW/731.

Cooper, R. G. (1993). Winning at New Products: accelerating the process from idea to launch. Reading: Addison-Wesley Publishing.

Cress, U., \& Fischer, G. (2017) Mass Collaboration with Social Software in TEL. In: Duval, E., Sharples, M., \& Sutherland, R. (Eds). Technology Enhanced Learning (pp. 59-67). Cham: Springer. 
Crossan, M.M., \& Apaydin, M. (2010). A Multi-Dimensional Framework of Organizational Innovation: A Systematic Review of the Literature. Journal of Management Studies, 47(6), 1154-1191. DOI: http://10.1111/j.1467-6486.2009.00880.x.

Dames, K. M. (2006). Social software in the library. 2006. Recuperado em 17 mar. 2016, de <http://www.Ilrx.com/features/socialsoftware.htm>.

He, W., \& Yan, G. (2013). Examining the use of social media in customer co-creation: a blog mining study. Proceedings of the Americas Conference on Information Systems, Chicago, IL, Estados Unidos, 19, 1-10.

Hidalgo, A., \& Albors, J. (2008). Innovation management techniques and tools: a review from theory and practice. R\&D Management, 38(2), 113-127. DOI: https://doi.org/10.1111/j.14679310.2008.00503.x.

Hinchcliffe, D. (2007). The state of enterprise 2.0. The enterprise web, 2.

Jue, A. L., Marr, J. A., \& Kassotakis, M. E. (2010). Mídias Sociais nas Empresas: Colaboração, Inovação, Competitividade e Resultados. São Paulo: Évora.

Khurana, A., \& Rosenthal, S. R. (1998). Towards holistic "front ends" in new product development. Journal of Product Innovation Management, 15(1), 57-74. DOI: https://doi.org/10.1016/S07376782(97)00066-0.

Machado, A. C. T. (2009). A ferramenta Google Docs: construção do conhecimento através da interação e colaboração. Revista Paidéi@, 2(1).

McAfee, A. (2010). Empresas 2.0: a força das mídias colaborativas para superar grandes desafios empresariais. São Paulo: Campus.

Miranda, M.A.S., Santos, E. M. (2015). Uso de softwares sociais para a gestão da inovação: estudo de caso em uma empresa de serviços. Anais do Congresso Latino-Iberoamericano de Gestão da Tecnologia, Porto Alegre, RS, Brasil, 16.

Nogueira, M.O., \& Oliveira, J.M. (2013). Da baleia ao ornitorrinco: contribuições para a compreensão do universo das micro e pequenas empresas brasileiras. Instituto de Pesquisa Econômica Aplicada IPEA - Radar № 25: tecnologia, produção e comércio exterior. Brasília: IPEA.

Organização para Cooperação e Desenvolvimento Econômico (2005). Manual de Oslo: diretrizes para coleta e interpretação de dados sobre inovação. 3. ed. Traduzido pela FINEP- Financiadora de Estudos e Projetos.

Peltola, T., \& Mäkinen, S. J. (2014). Influence of the adoption and use of social media tools on absorptive capacity in new product development. Engineering Management Journal, 26(3), 45-51. DOI: https://doi.org/10.1080/10429247.2014.11432019.

Pereira, R., Baranauskas, M. C., \& Silva, S. R. P. (2010). Softwares Sociais: Uma Visão Orientada a Valores. Proceedings of Symposium on Human Factors in Computing System, Belo Horizonte, MG, Brasil, 9, 149-158.

Perez, C. (2002). Technological Revolutions and Financial Capital: The Dynamics of Bubbles and Golden Ages. London: Elgar.

Piller, F., Vossen, A., \& Ihl, C. (2012). From social media to social product development: the impact of social media on co-creation of innovation. Swiss Journal of Business Research and Practice, 66(1), 728. DOI: http://dx.doi.org/10.5771/0042-059X-2012-1-7. 
Pugh, S. (1991). Total design: integrated methods for successful product engineering. Harlow: Addison Wesley.

Quandt, C. (2004). Inovação em clusters emergentes. Revista Com.Ciência, 57, 1-5.

Quevedo, L. A. (2007). Conhecer para participar da sociedade do conhecimento. In: Maciel, M.L., Albagli, S. (Orgs.). Informação e desenvolvimento: conhecimento, inovação e apropriação social (pp. 55-70), Brasília: IBICT, UNESCO.

Rantanen, T. (2005). The message is the medium: an interview with Manuel Castells. Global Media and Communication, 1(2), 135-147. DOI: https://doi.org/10.1177/1742766505054629.

Roberts, E. B. (1988). What We've Learned - Managing Invention and Innovation. Research-Technology Management, 31(1), 11-29. DOI: https://doi.org/10.1080/08956308.1988.11670497.

Rohmann, S., Heuschneider, S., \& Schumann, M. (2015). Social software for products development explorative study of influencing factors and related changes. Proceedings of Americas Conference on Information Systems, San Juan, USA, 21.

Rothwell, R. (1992). Successful industrial innovation: critical factors for the 1990s. R\&D Management, 22(3), 221-239. DOI: https://doi.org/10.1111/j.1467-9310.1992.tb00812.x.

Rozenfeld, H. et al. (2006). Gestão de Desenvolvimento de Produtos: uma referência para a melhoria do processo. São Paulo: Saraiva.

Song, L. Z. \& Song, M. (2010). The Role of Information Technologies in Enhancing R\&D-Marketing Integration: An Empirical Investigation. Journal of Product Innovation Management, 27(3), 382-401. DOI: https://doi.org/10.1111/j.1540-5885.2010.00723.x.

Teixeira, F. L. C., Hastenreiter Filho, H. N., Pires, A. M., \& Oliveira, S. R. G. (2011). Web 2.0 nas Empresas: a que será que se destina? GV Executivo, 10(2), 31-34. DOI:

http://doi.org/10.12660/gvexec.v10n2.2011.22831.

The World Bank Group (2014). World Development Indicators. Washington DC: The World Bank Data.

Thomas, R. J. (1993). New Product Development: managing and forecasting for strategic success. New York: John Wiley \& Sons.

Tidd, J., Bessant, J., \& Pavitt, K. (2005). Managing Innovation: Integrating Technological, Market and Organizational Change. 3.ed. Chichester: John Wiley \& Sons.

Tucker, J. M., Barczak, G., \& Hultink, E. J. (2014). Do social media tools impact the development phase? An exploratory study. Journal of Product Innovation Management, 31(S1), 18-29. DOI: http://onlinelibrary.wiley.com/doi/10.1111/jpim.12189/abstract.

Ulbricht, V. R., Gonzalez, R. D., Alves, M. M., Kuntz, V. H., \& Macedo, C. S. (2014). Ferramentas colaborativas aplicadas no ensino de geometria: proposta de utilização de uma rede social com atividades acessíveis. Educação Gráfica, 18(2), 80-96.

West, J. \& Gallagher, S. (2006). Challenges of open innovation: the paradox of firm investment in open-source software. $R$ \&D Management, 36(3), 319-331. DOI: https://doi.org/10.1111/j.14679310.2006.00436.x. 